$>$ Une révolution est actuellement en cours dans le domaine des maladies neuromusculaires avec l'arrivée de nouvelles thérapies. L'amyotrophie spinale (SMA ou spinal muscular atrophy) est parmi les maladies pionnières de ce bouleversement thérapeutique. Le premier traitement approuvé et mis sur le marché en Europe et aux États-Unis est un oligonucléotide antisens dénommé nusinersen et commercialisé par le laboratoire Biogen sous le nom de Spinraza ${ }^{\circledR}$. II a comme indication les SMA de types 1,2 et 3. La première injection de Spinraza ${ }^{\circledR}$ dans le cadre d'une ATU/EAP (Autorisation Temporaire d'Utilisation/Expanded Access Program ou programmes d'accès étendu) a été réalisée en France par le centre d'essais I-Motion. Les résultats des essais cliniques et des données de la littérature sur l'utilisation du nusinersen dans la SMA infantile sont discutés dans cette revue. Ces études rapportent une amélioration de la fonction motrice chez les patients SMA tous types confondus y compris les patients de type $3[1,2]$. Une administration précoce du traitement s'accompagne d'une meilleure réponse clinique. Une meilleure compréhension de l'hétérogénéité génétique et clinique devient indispensable dans le monitoring et le suivi à long terme de ces patients. <

\section{État des lieux}

La SMA est une des maladies neuromusculaires les plus fréquentes de l'enfant mais concerne également les adultes. C'est la cause génétique la plus fréquente de mortalité infantile [3]. Selon l'âge du début des premiers signes et le niveau moteur maximal atteint, la SMA est classée selon cinq types. La SMA de type 0 avec un début des signes dans la période anté- ou néonatale. La SMA de type 1 (ou maladie du Werdnig-Hoffmann) se caractérise par un début des premiers signes

\section{Les \\ oligonucléotides anti-sens dans Ia SMA \\ Retour d'expérience et données de la littérature}

Elena Gargaun

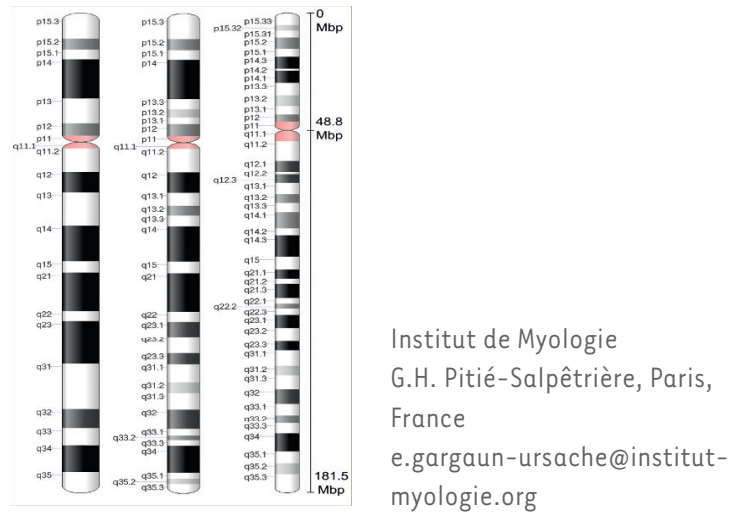

avant l'âge de 6 mois et une durée de vie sans traitement inférieure à 9-18 mois. Certains patients, dits de type lbis, survivent plus de 18 mois mais n'acquièrent jamais la station assise indépendante. Pour la SMA de type 2, les premiers signes apparaissent après 6 mois et les enfants sont capables de se tenir assis de façon indépendante pendant plus d'une minute. Pour la SMA de type 3, (ou maladie de KugelbergWelander) le début est plus tardif, avec un risque important de perte progressive d'une marche autonome initialement acquise. Enfin la SMA de type 4 est caractérisée par un début des symptômes à l'âge adulte [4].

La SMA a une incidence de l'ordre de $1 / 10000$ naissances avec une fréquence de porteurs sains hétérozygotes de 1/50 [5]. C'est une maladie autosomique récessive due à des mutations dans le gène SMN1 (survival motor neuron 1) localisé sur le chromosome 5q13.2 [6]. Elle se caractérise par une faiblesse musculaire proximale suite à la dégénérescence progressive des motoneurones de la corne antérieure de la moelle épinière et du tronc cérébral. La prévalence communément admise pour la France (enquête SMA-Flash, Filnemus) tourne autour de 1200 patients SMA dont $8 \%$ de type $1,47 \%$ de type $2,42 \%$ de type 3 et $2 \%$ de type 4 [7].

\section{Données génétiques dans la SMA}

Dans $95 \%$ des cas environ, la SMA est due à une délétion homozygote, soit de l'exon 7 , soit des exons 7 et 8 , du gène SMNI (5q12.2-q13.3) codant la protéine de survie du motoneurone (SMN). Situé en région 
centromérique, le gène $S M N 2$, est capable de produire la protéine $S M N$, mais celle-ci est non fonctionnelle dans $90 \%$ des cas à cause d'une erreur d'épissage. La seule différence entre les deux gènes réside dans la substitution $C->T$ qui génère un épissage alternatif de l'exon 7 . Les $10 \%$ de la protéine SMN produite par le gène SMN2 sont insuffisants pour maintenir la survie et le fonctionnement normal des motoneurones [6]. Le rôle de la protéine SMN n'est pas encore complètement connu. Elle est associée avec la biogenèse de la protéine nucléaire ribonucléique (snRNP) et le métabolisme des ARN messagers [8]. De plus, les données de la littérature suggèrent qu'en son absence survient une dégénérescence des neurones sensitifs et de la jonction neuromusculaire [8].

\section{Hétérogénéité phénotypique des patients atteints de SMA}

Le nombre de copies du gène SMN2 est inversement corrélé avec le phénotype clinique. II est important de souligner que le nombre de transcrits SMN2 n'est pas forcement identique au nombre de copies de SMN2 à cause de la variabilité de la régulation de la transcription inter individus. Des données récentes ont rapporté des polymorphismes du SMN2 en tant que modificateurs géniques [9 -11]. Malgré cela, aux États-Unis, le groupe de travail multidisciplinaire SMA NBS a émis les recommandations d'algorithme thérapeutique basé sur le nombre des copies de SMN2 pour les patients SMA diagnostiqués par dépistage néonatal [12]. Il est important de connaître et de comprendre au mieux l'hétérogénéité génique et clinique des patients pour le diagnostic et le pronostic clinique mais également pour comprendre et quantifier la réponse thérapeutique.

\section{Options thérapeutiques}

Diverses thérapies incluant des oligonucléotides antisens (ASO) et des petites molécules ont été mises au point pour interférer avec l'épis- sage alternatif de SMN2 augmentant la transcription de I'ARN messager et par voie de conséquence de la protéine SMN produite. Parmi ces nouvelles thérapies, Spinraza ${ }^{\circledR}$ (nom commercial du nusinersen) s'administre en injections intrathécales, avec une dose fixe de $12 \mathrm{mg}$ par administration pour tous les patients tout âge confondu $[1,2]$. Le traitement doit débuter le plus tôt possible après le diagnostic, avec quatre doses de charge aux jours $0,14,28$ et 63 . Une dose d'entretien doit ensuite être administrée tous les quatre mois.

D'autres options thérapeutiques telles que l'injection unique d'AAV par voie intraveineuse pour traiter les enfants SMA de moins de deux ans (Zolgensma ${ }^{\circledR}$ ) et les petites molécules (comme la flunarizine) ne seront pas abordées dans cette revue.

\section{Les essais cliniques nusinersen dans la SMA}

Les résultats précliniques encourageants et des études en ouvert ont permis la réalisation des essais cliniques de phase 3 pour évaluer l'efficacité thérapeutique du nusinersen chez les patients SMA de types 1 et 2 (Tableau I).

En Europe, le nusinersen a été administré initialement dans le cadre d'un programme d'autorisation temporaire d'utilisation (ATU/EAP). Certains résultats du suivi de ces patients seront discutés ci-dessous.

\section{SMA de type 1}

Dans l'essai clinique multicentrique ENDEAR (CS3B) de phase 3 contrôlé en double aveugle ont été inclus

\begin{tabular}{|c|c|c|c|c|c|}
\hline & CS3A & $\begin{array}{l}\text { Endear } \\
\text { (CS3B) }\end{array}$ & CS2/CS12 & Cherish CS4 & Nurture \\
\hline Sponsor & Ionis & Biogen & Ionis & Biogen & Biogen \\
\hline Design de l'étude & En ouvert & Contrôlé & En ouvert & Control vs procédure placebo & Contrôlé ou en ouvert \\
\hline N & 20 & 123 & $28-58$ & 126 & 17 \\
\hline Critères d'inclusion & $\begin{array}{l}<7 \text { mois } \\
\text { SMAl }\end{array}$ & $\begin{array}{l}<7 \text { mois } \\
\text { SMN } 2=2 \\
\text { SMAl }\end{array}$ & $\begin{array}{l}2-15 \text { ans } \\
\text { SMA } 2,3\end{array}$ & $\begin{array}{l}2-12 \text { ans } \\
\text { SMA2 }\end{array}$ & $\begin{array}{l}\text { Présymptomatique } \\
\text { Exam neuro NL } \\
\text { CMAPl mV }\end{array}$ \\
\hline Evaluations & $\begin{array}{l}\text { Survie/resp. } \\
\text { HINE2 } \\
\text { CHOP INTEND } \\
\text { CMAP }\end{array}$ & $\begin{array}{l}\text { Survie/resp. } \\
\text { HINE2 } \\
\text { CHOP INTEND } \\
\text { CMAP }\end{array}$ & $\begin{array}{l}\text { HFMSE } \\
\text { 6MWT } \\
\text { RULM }\end{array}$ & $\begin{array}{l}\text { HFMSE } \\
\text { RULM }\end{array}$ & $\begin{array}{l}\text { Survie/resp. } \\
\text { HINE2 } \\
\text { CMAP }\end{array}$ \\
\hline
\end{tabular}

Tableau I. Essais cliniques avec traitement par Spinraza ${ }^{\circledR}$ (Nusinersen) (adapté de Claudia A. Chiriboga [13]). AS : amyotrophie spinale ; N : nombre des patients ; NL : normal ; exam neuro : examen neurologique; resp. : ventilation respiratoire permanente, trachéostomie ou $\geq 16$ h de ventilation ; HINE2 : The Hammersmith Infant Neurological Examination; CHOP INTEND : The Children's Hospital of Philadelphia Infant test for Neuromuscular Disorders; HFMSE : Hammersmith Functional Motor Scale Expanded ; 6MWT : test de marche de 6 min ; RULM : Revised Upper Limb Module ; CMAP : Compound Muscle Action Potential. 
121 enfants avec SMA de type 1, tous avec deux copies de SMN2 et âgés de moins de 7 mois à la visite de sélection. L'étude a été arrêtée prématurément au bout de 13 mois suite aux résultats d'analyse intermédiaire qui ont montré une réponse positive significative du test HINE (Hammersmith Infant Neurological Examination) chez les patients sous traitement. Les patients ont été tous inclus dans le groupe traité et à la fin de l'étude les survivants ont été inclus dans l'étude CS1l [1].

\section{SMA de type 2}

Dans l'essai clinique CHERISH (CS4) de phase 3 multicentrique, contrôlé en double aveugle, 126 enfants ont été inclus. Les patients inclus présentaient une SMA de type 2 (2 à 12 ans), sans scoliose sévère ou rétractions articulaires avec une randomisation de 2:1. L'analyse intermédiaire à 15 mois a mis en évidence une différence significative du score HFMSE (Hammersmith Functional Motor Scale Expanded) entre le groupe traité versus contrôle, ce qui a conduit à un arrêt prématuré de l'étude et la mise en place d'une phase d'extension CS11 [2].

\section{Patients présymptomatiques}

Dans l'étude NURTURE (CS5/SM201), 17 patients ont été inclus avec diagnostic prénatal ou à la naissance de SMA. La majorité des patients avait deux copies de SMN2 (12/17). Tous les patients traités ont survécu sans aide ventilatoire et ils continuent de progresser dans leur développement psychomoteur.

\section{Patients SMA de type $1 / 1$ bis tout âge confondu traités sous ATU}

Plusieurs études récentes ont évalué l'évolution clinique chez des patients SMA de types 1 et lbis traités par Spinraza ${ }^{\circledR}$ dans le cadre de l'ATU (EAP) $[13,14]$. En comparaison avec les essais cliniques, les patients inclus dans ces cohortes étaient d'âges variables et avec une durée d'évolution de la maladie plus longue et un début de traitement parfois bien au-delà de la limite des 6 mois choisie lors des essais cliniques. Globalement, les évaluations motrices chez les patients avec un début de traitement précoce avant 7 mois ont été comparables aux résultats rapportés dans les essais cliniques. L'étude italienne rapporte une amélioration après 12 mois de traitement des résultats de CHOP INTEND (Children's Hospital of Philadelphia Infant Test Neuromuscular Disorder scale) (moyenne 5,46 points) et dans moins de $15 \%$ des cas une aggravation dans cette cohorte [9]. Dans une étude allemande, il a été proposé aux parents de donner leur appréciation sur l'évolution sur le plan moteur chez les enfants SMA de type lbis traités. Dans 93,4\% de cas, la réponse au traitement a été jugée favorable par les familles. Cette appréciation n'était toutefois pas significativement corrélée avec les résultats du CHOP-INTEND [13]. Par ailleurs, il est encore difficile d'établir une corrélation des progrès identifiés avec la CHOP-INTEND et leur traduction au quotidien chez les patients avec SMA de type 1. Cette échelle reste néanmoins un outil précieux de suivi des patients SMA. Après la mise sur le marché du nusinersen, la question de poursuivre ce type de traitement lourd et onéreux se pose pour les patients avec peu ou avec des progrès modestes. Dans l'étude italienne, moins de $7,5 \%$ des patients ont arrêté le traitement [13]. Le suivi des patients, issus de différents pays de la Communauté Européenne, a mis en évidence une différence de prise en charge entre centres et entre pays.

\section{Conclusion}

Spinraza ${ }^{\circledR}$ (nusinersen) est le premier traitement approuvé et mis sur le marché en Europe et aux ÉtatsUnis. La première injection de Spinraza ${ }^{\circledR}$ dans le cadre d'une ATU a été réalisée en France (Institut I-Motion, Hôpital Trousseau). Le traitement des patients SMA de type 1 est maintenant devenu possible dans de nombreux centres hospitaliers en France. Spinraza ${ }^{\circledR}$ est aussi disponible actuellement pour les patients SMA de types 2 et 3 . L'intérêt du traitement serait de préserver les acquisitions des patients et d'empêcher la progression de la maladie. Chez les patients avec la SMA de type lbis, l'indication au cas par cas semblerait être une attitude plus prudente suite aux résultats modestes chez ces patients dans les cohortes traitées initialement dans le cadre d'une ATU. Les données précliniques et les résultats des essais thérapeutiques mettent en évidence l'existence d'une fenêtre d'administration thérapeutique pour une meilleure réponse clinique. Ces données ouvrent le débat autour du dépistage néonatal pour la mise en place du traitement dans les délais recommandés par les essais cliniques. Un groupe de travail ad hoc est désormais en place sous l'égide de la filière FILNEMUS rassemblant les professionnels de santé concernés et les représentants de patients. Les études publiées sur les cohortes des patients traités sous ATU en Europe ont mis en évidence une large hétérogénéité dans la prise en charge de ce type de

\section{Leçons apprises}

- Diagnostic urgent avec un choix de traitement disponible.

- Nécessité d'accompagnement des familles devant ce choix cornélien.

- Nécessité d'organisation locale pour la mise en place précoce du traitement.

- Création d'un registre pour la surveillance de l'évolution des patients traités.

- Plusieurs choix thérapeutiques bientôt disponibles.

- Nécessité d'ouvrir le débat autour du dépistage néonatal de la SMA.

- Implémentation des standards de soins.

- Hétérogénéité génique et clinique des patients. 
patients, avec la présence dans certains cas de l'assistance ventilatoire et/ou des différences dans le type de rééducation motrice et la prise en charge nutritionnelle. Ces études soulignent largement l'intérêt de l'implémentation des standards de soins concomitante à toute démarche thérapeutique. $\diamond$

Antisense oligonucleotides in SMA: lessons learned and literature data

\section{LIENS D'INTÉRÊT}

E. Gargaun a travaillé en tant qu'investigateur dans les essais cliniques sur le nusinersen et a réalisé les premières injections dans le cadre de l'ATU en France à I-Motion.

\section{RÉFÉRENCES}

1. Finkel RS, Chiriboga CA, Vajsar J, et al. Treatment of infantile-onset spinal muscular atrophy with nusinersen: a phase 2, open-label, dose-escalation study. Lancet 2016 ; $388: 3017-26$.

2. CA Chiriboga, Swoboda KJ, Darras BT, et al. Results from a phase 1 study of nusinersen (ISIS$\operatorname{SMN}(\mathrm{Rx}))$ in children with spinal muscular atrophy. Neurology Mar $2016 ; 86: 890-7$.

3. Darras BT. Spinal muscular atrophies. Pediatr Clin North Am $2015 ; 62$ : 743-66.

4. Kolb SJ, Coffey CS, Yankey JW, et al. Natural history of infantile-onset spinal muscular atrophy. Ann Neurol 2017 ; 82 : 883-91.

5. Verhaart IEC, Robertson A, Wilson IJ, et al. Prevalence, incidence and carrier frequency of 5q-linked spinal muscular atrophy : a literature review. Orphanet J Rare Dis $2017 ; 12: 124$

6. Lefebvre $S$, Bürglen L, Reboullet $S$, et al. Identification and characterization of a spinal muscular atrophy-determining gene. Cell $1995 ; 80: 155-65$.
7. Urtizberea JA, Daidj F. Réseau Filnemus. Med Sci (Paris) 2018 ; 34 (hs2) : 32-4.

8. Kariya S, Obis T, Garone C, et al. Requirement of enhanced surviva motoneuron protein imposed during neuromuscular junction maturation. J Clin Invest $2012 ; 124$ : 785-800.

9. Bernal S, Alías L, Barceló MJ, et al. The c.859G>C variant in the SMN2 gene is associated with types II and III SMA and originates from a common ancestor. Med Genet $2010 ; 47: 640-2$.

10. Vezain M, Saugier-Veber P, Goina $\varepsilon$, et al. A rare SMN2 variant in a previously unrecognized composite splicing regulatory element induces exon 7 inclusion and reduces the clinical severity of spinal muscular atrophy. Hum Mutat $2010 ; 31:$ : $11110-25$

11. Wu X, Wang SH, Sun J, et al. A-44G transition in SMN2 intron 6 protects patients with spinal muscular atrophy. Hum Mol Genet 2017 ; 26 : 2768-80.

12. Glascock J, Sampson J, Haidet-Phillips A, et al. Treatment algorithm for infants diagnosed with spinal muscular atrophy through newborn screening. J Neuromuscul Dis $2018 ; 5: 145-58$.

13. Pane M, Coratti G, Sansone VA, et al. Nusinersen in type 1 spinal muscular atrophy: twelve-month real-world data. Ann Neurol 2019; 86 : 4 43-51.

14. Pechmann A, Langer T, Schorling D, et al. Evaluation of children with SMA type 1 under treatment with Nusinersen within the expanded access program in Germany. J Neuromuscul Dis $2018 ; 5: 135-43$.

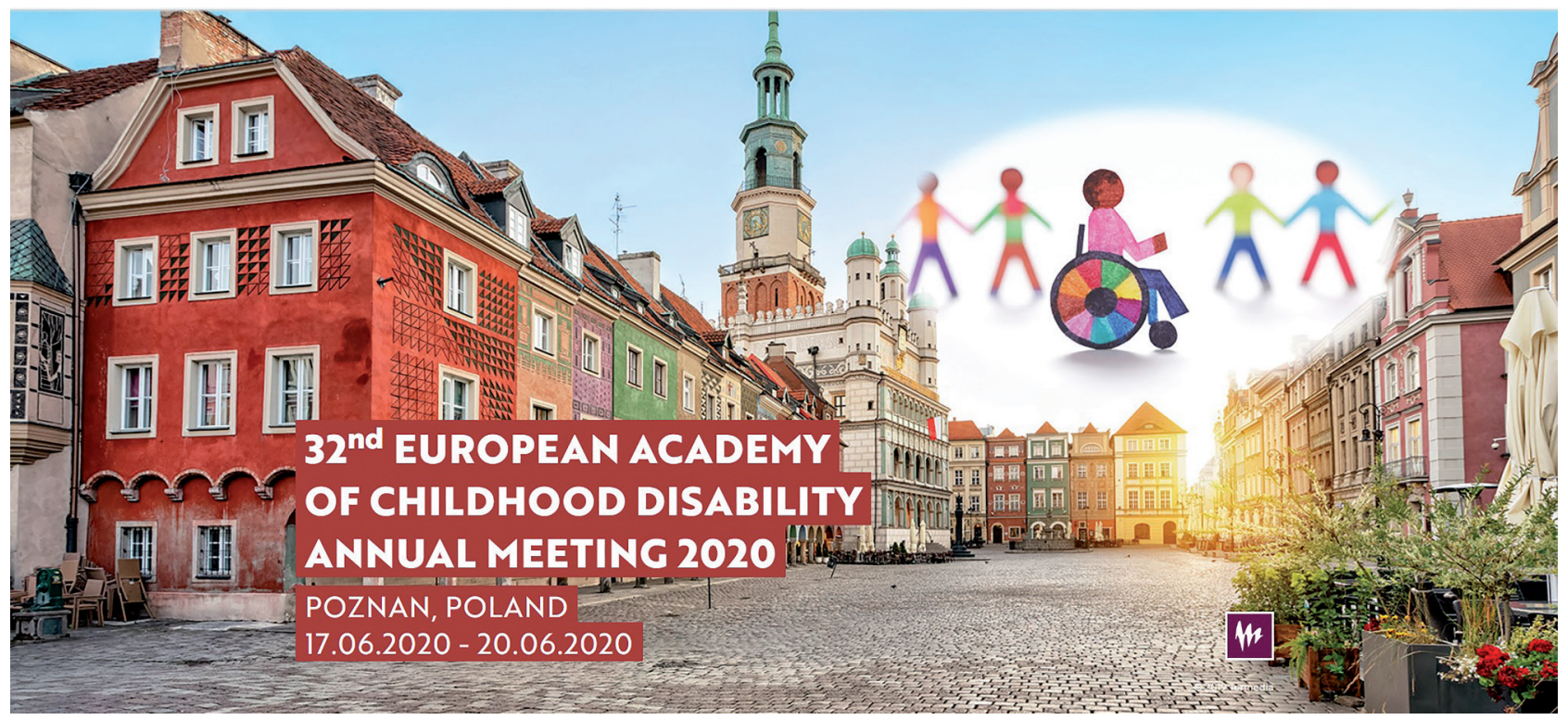

\section{Retrouvez toutes les Actualités de la Myologie}

sur les sites de :

TIRÉS À PART

દ. Gargaun 